\title{
Investigation of Energy Dissipation and Droplets Dispersion in a Horizontal Pulsating Resonance Apparatus
}

\author{
Lyazzat M. Satayeva ${ }^{1}$ \\ Rufat Sh. Abiev² \\ Abdugani M. Azimov ${ }^{3}$ \\ Arsen Ye. Ortayev ${ }^{4}$ \\ Igor V. Yaroschik ${ }^{5}$ \\ 1,3,4 M. Auezov South Kazakhstan State University, Shymkent, Kazakhstan \\ ${ }^{2,5}$ St. Petersburg State Technological Institute (Technical University), St. Petersburg, Russian Federation
}

Doi:10.5901/jesr.2016.v6n1p147

\begin{abstract}
The paper describes results of comparative experimental investigations to determine quality of droplets dispersion, as an indicator of efficiency of introduced into the apparatus energy and determine an optimal oscillation frequency of the heterogeneous media in the apparatus. The research is based on the fact that better droplet breakup occurs at the resonant mode, as well as a power, introduced into the system, more completely dissipated in a mixture. Increase in the apparatus reliability is provided by a conventional separation of a liquid's oscillating mass into the even-numbered amount of equivoluminar communicating sections, in organization of antiphase pulsations in them by oppositely directed towards the pulsating chamber motions of the liquid in the neighboring to it sections. According to the developed by us theory, there is a possibility to change the power, supplied on the dispersion of dispersed phase' droplets into the continuous one by application of the droplets' samples maximal diameter values on a graph of diameters against the energy dissipation. By the research results, it may be concluded that principles for generation of oscillation, realized in a horizontal pulsating resonance apparatus, have great perspectives for application in the modern equipment.
\end{abstract}

Keywords: droplets dispersion, horizontal pulsating resonance apparatus, energy saving apparatus, pulsating mixing.

\section{Introduction}

In connection with the aggravation of the energy and raw materials problems in recent years, worldwide increased interest to reduce energy and material resources costs with a concomitant increase of beneficial effects, including water treatment (Sarkisov (2000)).

Methods to improve the characteristics of the chemical and technological equipment can be divided into two classes: 1) optimization of existing apparatuses, installations and production on the basis of formal mathematical methods; 2) search for the most effective for the process of physical impacts, including the adaptation of the geometric shape of vehicles and their components. Abiev (2008) has discussed the general principles of process intensification in the examples of pulsating devices. Most consistently, in terms of energy conversion efficiency, the idea of energy consumption reducing in the manufacturing processes was implemented by Dolinskii and Ivanitskii (2008) and Ganiev and Ukrainskii (2011).

Examples of implementation of the described in (Abiev (2008)) principles in the apparatuses for different processes: reducing the time of wastewater treatment by JSC "Akron" at using pulsatile flow type apparatus (Abiev (2012)), fine mechanical treatment of water from mechanical impurities with a density close to the density of water (Yaroschik and Abiev (2013)), use of advanced thin- layer settling tanks for wastewater treatment (Abiev, Azimov, Shakirov (2013)), use of membrane device pulsation type (Azimov, Abiev, Shakirov, Zhantasov (2014)), vacuum degassing of water using a vortex jet device (Abiev, Vasilev, Doilnitsyn (2015)), the pulsation membrane device for desalination of natural and industrial waters with periodic discharge of sludge thickening (Azimov, Abiev, Zhantasov, Sataeva, Azimova, Zerhatbek (2015)). 
Horizontal pulsating resonance apparatus (HPRA) is new kind of energy-saving equipment and can be used for a wide range of problems: dispersion, intensification of heat and mass transfer, including extraction, purification of membranes in membrane devices, dissolution of heavy precipitation, liquid extraction process.

Furthermore, HPRA is a convenient model for study of vibrations in the apparatus where pulsations are created for intensification various hydromechanical and mass transfer processes: for dispersing the droplets in the extraction purification process, for cleaning the surface of membrane devices, for rapid dissolution of flocculants and other solid reactants in wastewater.

The aim of presented research paper is to determine the quality of the droplets dispersion, as an indicator of the energy efficiency input into the apparatus and determine the optimal oscillation frequency of environment in the apparatus. This frequency is determined by the oscillation frequency of the stock 6 (see Fig. 1), which performs reciprocating motions and is connected to the driving force of the working environment vibrations, designed as a silphon, forming with a pulsation apparatus body gas-filled pulsation chamber. The applications of our study were: 1) to find out the possibility to use pulsations for fine dispersion of droplets and further to carry out chemical reactions in the apparatus, e.g. production of biodiesel or extraction of aromatics from fuels (mineral diesel and petrol). 2) to show the resonance character of pulsations and its effect on the droplet size; 3 ) to prove experimentally new principle to predict the energy dissipated in the apparatus.

It is known from the papers of Ganiev and Ukrainskii (2011)., Dolinskii and Ivanitskii (2008), that strict non steady state impact on the heterogeneous media could have much more effect than soft impact. Therefore we have aimed our work to check the effectiveness of pulsations in general and resonance pulsations particularly in the process of droplet dispersion. Moreover, the sizes of droplets could serve as an indicator of effectiveness of energy transformation in different apparatuses.

\section{Experimental}

Starting components (vegetable oil (in a volume of 1 liter), alkali solution $(\mathrm{KOH})$ and methanol (in a volume of $150 \mathrm{ml}$ per liter of oil)) are fed into the apparatus through a funnel 8 . Monitoring of process progress is going through dimensional glasses 2. The experiments are carried out at different frequency of stock oscillation to determine the optimal frequency of stock oscillation. It is assumed that with an estimated value of the resonant frequency, the reaction time will be minimal. With the heating elements 3 and the temperature controller sensor during each experiment is possible to maintain a certain temperature in the apparatus.

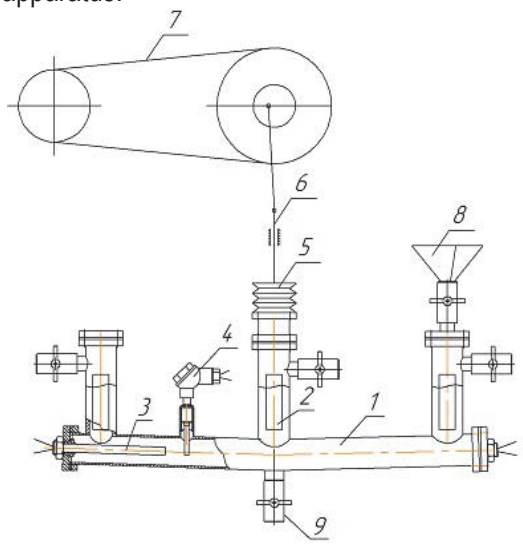

1 - body; 2 - dimensional glass; 3 - heating element; 4 - thermocouple; 5 - silphon; 6 - stock; 7 - electromechanical drive; 8 - funnel; 9 - tap.

Figure 1: Experimental setup of the HPRA

Improving the reliability of the apparatus is ensured by a conditional division of the oscillating liquid mass on an even number of coextensive communicating sections at the organization antiphase oscillations due to the opposite direction to the pulsation chamber fluid flows in the adjoining sections. Dynamic loads resulting from the oscillating masses in each section, mutually balance each other out, i.e. fluid fluctuations in the sections, arranged symmetrically to pulsation 
chambers, are antiphase, and fluid mass in them are equal. Therefore, the dynamic load on the foundation is fully offset, which increases the reliability of the apparatus.

At startup of the oscillator are driven fluctuations in the pulsation chambers which transmit fluid in each section. Fluctuations in the sections occur antiphase. Due to the balance of fluctuating fluid masses, it is possible to carry out high-intensity vibrations in the apparatus, as well as resonance oscillations. At the oscillation frequency of the fluid given by the generator close to the natural frequency of the system fluid in section - the gas in the resonance chamber there are resonant oscillations of the fluid leading to a sharp increase in the amplitude of fluctuations and intense turbulence of the fluid and its mixing, thereby significantly increases the efficiency of the process due to the powerful dynamic effects on the treated system.

The research is conducted on the basis that at resonant mode is the best crushing of drops and power that is injected into the system more fully dispersed in the working mixture.

In this experiment tap water was used as the solid phase, media temperature was $16^{\circ} \mathrm{C}$. Dispersed phase was the sunflower refined oil, the temperature of the starting material was $21^{\circ} \mathrm{C}$.

Temperature of the mixture after the establishment of a thermocouple was $18,3^{\circ} \mathrm{C}$. The water volume in the reactor was $1.14 \mathrm{I}$, injected volume of sunflower oil was $1 \%$ from weight of the mixture, namely $11 \mathrm{ml}$. The oil supply was made to the central pipe through side branch for fixing the tube in an amount of $6 \mathrm{ml}$, and through the hopper of the left nozzle in an amount of $5 \mathrm{ml}$. Then installation was launched. There were carried out 5 experiments at different frequencies 11.1, 12,035, 12.67, 14.183, $14.818 \mathrm{~Hz}$.

The duration of each experiment was 10 minutes, and then sampling was performed in a plastic beaker from which via a syringe the emulsion was selected and was placed on a microscope slide for photographing. After each experiment, the reactor was flushed with the washing liquid. For each of the test samples some photos were obtained, the most successful of them have been treated. Photos are shown in Figures 2-6.

\section{Results and Discussion}

Photos treatment consisted in the following. Previously zooming scale of microscope was held using calipers. $1 \mathrm{~mm}$ caliper scale corresponds to 7 large scale division of the microscope eyepiece. Photos treatment was performed using the program Kompas 3D. On the $1 \mathrm{~mm}$ a length corresponding to 7 bars scale ocular, in a program the scale was chosen so that the actual length of $1 \mathrm{~mm}$ corresponded to $500 \mathrm{~mm}$ in length, i.e. scale was 500:1. Then the drops were encircled by circles of a certain size, it has been estimated the total number of circles of each diameter, and the end results were recorded in the table.

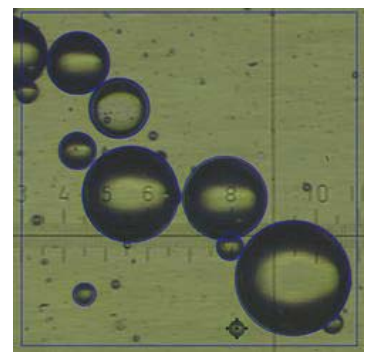

Figure 2: Photos of drops at a frequency of $11.1 \mathrm{~Hz}$ pulsations

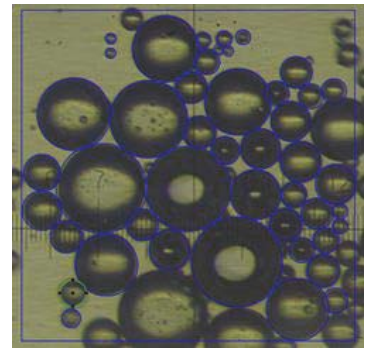

Figure 3: Photos of drops at a frequency of $12,035 \mathrm{~Hz}$ pulsations 
Figure 4: Photos of drops at a frequency of $12.67 \mathrm{~Hz}$ pulsations
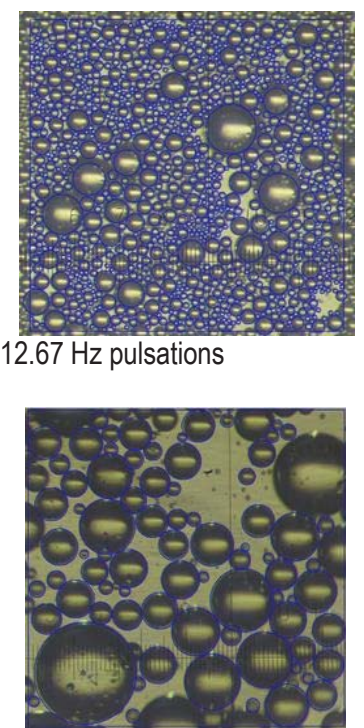

Figure 5: Photos of drops at a frequency of $14,183 \mathrm{~Hz}$ pulsations

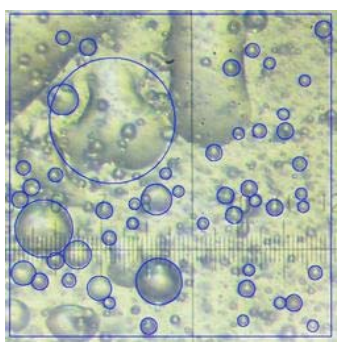

Figure 6: Photos of drops at a frequency of $14,818 \mathrm{~Hz}$ pulsations

Also converted values of the droplet diameters were recorded in the table, these values were used for further calculations. All the results of photos treatment are shown in Tables 1-5.

As seen from the photos, as it approaches the resonance frequency (the frequency of $12.67 \mathrm{~Hz}$ in the stock) size of the oil droplets is reduced - it can be clearly seen in Figure 4, which shows the most complete dissipation of energy in the work environment. Then, with increasing distance from the resonance point, the droplet size increases again.

Table 1: Results of photo processing at a frequency of $11.1 \mathrm{~Hz}$

\begin{tabular}{|c|c|c|}
\hline circle diameter, $m m$ & droplet diameter, $m m$ & $\Delta$ Ni, $p c s$ \\
\hline 28 & 0.056 & 1 \\
\hline 34 & 0.068 & 1 \\
\hline 40 & 0.08 & 1 \\
\hline 56 & 0.112 & 1 \\
\hline 90 & 0.18 & 1 \\
\hline 94 & 0.188 & 1 \\
\hline 126 & 0.252 & 1 \\
\hline 145 & 0.29 & 1 \\
\hline 172 & 0.344 & 1 \\
\hline \multicolumn{2}{|c|}{ The total number of drops, $N=\sum \Delta N_{i}=9 \mathrm{pcs}$} \\
\hline
\end{tabular}


Table 2: Results of photo processing at a frequency of $12,035 \mathrm{~Hz}$

\begin{tabular}{|c|c|c|}
\hline circle diameter, $\mathrm{mm}$ & droplet diameter, $\mathrm{mm}$ & $\Delta$ Ni, pcs \\
\hline 16 & 0,032 & 4 \\
\hline 23 & 0,046 & 5 \\
\hline 28 & 0.056 & 1 \\
\hline 38 & 0.076 & 8 \\
\hline 42 & 0.084 & 2 \\
\hline 48 & 0.096 & 7 \\
\hline 55 & 0.11 & 1 \\
\hline 60 & 0.12 & 5 \\
\hline 76 & 0.152 & 1 \\
\hline 94 & 0.188 & 2 \\
\hline 100 & 0.2 & 2 \\
\hline 110 & 0.22 & 1 \\
\hline 117 & 0.234 & 1 \\
\hline 128 & 0.256 & 2 \\
\hline 136 & 0.272 & \\
\hline \multicolumn{2}{|c|}{ The total number of drops, $N=\sum \Delta N_{i}=43 \mathrm{pcs}$} \\
\hline \multicolumn{2}{|l}{} \\
\hline
\end{tabular}

Table 3: Results of photo processing at a frequency of $12,67 \mathrm{~Hz}$

\begin{tabular}{|c|c|c|}
\hline circle diameter, $m m$ & droplet diameter, $\mathrm{mm}$ & $\Delta \mathrm{Ni}, \mathrm{pcs}$ \\
\hline 7 & 0.014 & 265 \\
\hline 10 & 0.02 & 178 \\
\hline 13 & 0.026 & 121 \\
\hline 16 & 0.032 & 157 \\
\hline 20 & 0.04 & 49 \\
\hline 23 & 0.046 & 54 \\
\hline 29 & 0.058 & 25 \\
\hline 34 & 0.068 & 6 \\
\hline 38 & 0.076 & 7 \\
\hline 42 & 0.084 & 1 \\
\hline 52 & 0.104 & 2 \\
\hline 64 & 0.128 & 1 \\
\hline 80 & 0.16 & \\
\hline \multicolumn{2}{|c|}{ The total number of drops, $N=\sum \Delta N_{i}=869 \mathrm{pcs}$} \\
\hline
\end{tabular}

Table 4: Results of photo processing at a frequency of $14,18 \mathrm{~Hz}$

\begin{tabular}{|c|c|c|}
\hline circle diameter, $\mathrm{mm}$ & droplet diameter, $\mathrm{mm}$ & $\Delta \mathrm{Ni}, \mathrm{pcs}$ \\
\hline 14 & 0.028 & 4 \\
\hline 17 & 0.034 & 8 \\
\hline 22 & 0.044 & 12 \\
\hline 26 & 0.052 & 5 \\
\hline 29 & 0.058 & 3 \\
\hline 32 & 0.064 & 5 \\
\hline 36 & 0.072 & 1 \\
\hline 42 & 0.084 & 5 \\
\hline 48 & 0.096 & 7 \\
\hline 52 & 0.104 & 7 \\
\hline 58 & 0.116 & 5 \\
\hline 60 & 0.12 & 3 \\
\hline 68 & 0.136 & 3 \\
\hline 74 & 0.148 & 1 \\
\hline 76 & 0.152 & 2 \\
\hline 88 & 0.176 & 3 \\
\hline 100 & 0.2 & 1 \\
\hline 162 & 0.324 & 1 \\
\hline
\end{tabular}


Table 5: Results of photo processing at a frequency of $14,818 \mathrm{~Hz}$

\begin{tabular}{|c|c|c|}
\hline circle diameter, $m m$ & droplet diameter, $m m$ & $\Delta N i, \mathrm{pcs}$ \\
\hline 18 & 0.036 & 18 \\
\hline 22 & 0.044 & 8 \\
\hline 26 & 0.052 & 16 \\
\hline 38 & 0.076 & 2 \\
\hline 44 & 0.088 & 1 \\
\hline 48 & 0.096 & 2 \\
\hline 72 & 0.144 & 1 \\
\hline 90 & 0.18 & 1 \\
\hline 195 & 0.39 & \\
\hline \multicolumn{2}{|c|}{ The total number of drops, $N=\sum \Delta N_{i}=50 \mathrm{pcs}$} \\
\hline
\end{tabular}

For all 5 different frequencies were calculated average linear $\left(\delta_{10}\right)$, average of the surface $\left(\delta_{20}\right)$, weight average $\left(\delta_{30}\right)$ diameters, as well as to clarify the specific surface area and for further calculations was calculated Sauter mean diameter $\left(\delta_{32}\right)$.

For comparison of the average diameters was designed a schedule, which showed the dependence of the average diameters $\delta_{10}, \delta_{20}, \delta_{30}, \delta_{32}$ from the HPRA's stock frequency oscillation.

The values of the distribution functions were calculated on the linear dimensions, surface and weight, as well as the calculation of average diameters $\delta_{10}, \delta_{20}, \delta_{30}, \delta_{32}$ for the resonant stock frequency oscillation $(12.67 \mathrm{~Hz})$.

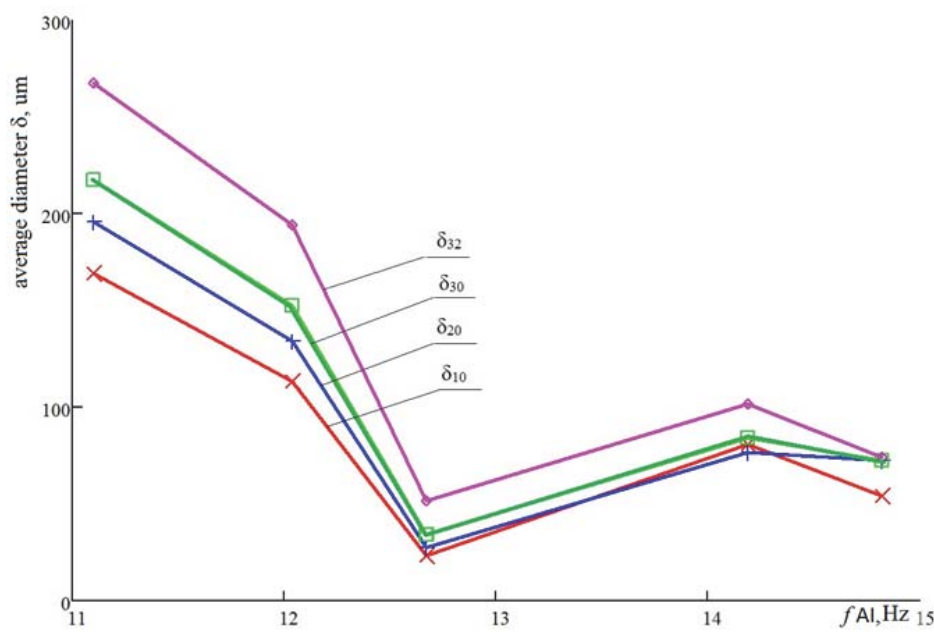

$\delta_{10}$ - average linear droplet diameter; $\delta_{20}$ - average droplet diameter over the surface; $\delta_{30}$ - mass median dropletdiameter; $\delta_{32}$ - Sauter mean diameter of drops.

Figure 7: The dependence of the mean droplet diameter from the oscillation frequency of the pulsator

First consider a number of diameters obtained by photos processing into intervals 0-20, 20-40, 60-80, 80-100, 100-140, 140-180 microns. Then you need to sort drops of different fractions respectively predetermined intervals. $\Delta \delta-$ the difference between the values of the interval boundaries $\delta i+1$ and $\delta i$.

When processing a function $f(\delta)$ was used, as it is more clearly reflects the dispersed properties of the particles.

Table 6: The total number of drops for the respective operating frequency of the pulsator

\begin{tabular}{|c|c|c|c|c|c|}
\hline $\mathrm{f}, \mathrm{Hz}$ & 11.1 & 12.035 & 12.67 & 14.183 & 14.818 \\
\hline $\mathrm{N}, \mathrm{pcs}$ & 9 & 43 & 869 & 76 & 50 \\
\hline
\end{tabular}


The density function of the droplet distribution on size is:

$$
f_{N i}=\frac{\Delta N_{i}}{N \cdot \Delta \delta i},
$$

where: $\Delta \mathrm{N}_{\mathrm{i}}-$ number of particles of corresponding fraction

$\mathrm{N}$ - total number of particles

$\Delta \delta i$ - size of particles of corresponding fraction.

Similarly is calculated density function of the droplet mass distribution on the size:

$$
f_{M}=\frac{\Delta M}{M \cdot \Delta \delta}
$$

For the interval of $0-20 \mathrm{~mm}$, the formula for the value of the distribution function on the linear dimension will be (2):

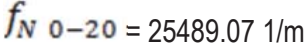

Similarly was carried out calculation for the rest size intervals.

Assuming that the oil droplets have a spherical shape, will find their area and weight in the range of sizes, based on the following relationships.

The following dependence was used for calculation of the drops mass:

$m=\frac{\rho \pi \delta^{3}}{6}$

If we integrate the expression (4), it is not difficult to obtain:

$\frac{\rho \pi}{6} \int \delta^{3} d \delta=\frac{\rho \pi \delta^{4}}{24}$

For calculation the drops area was used:

$s=\rho \pi \delta^{2}$

If we integrate the expression (6), it is easy to obtain:

$\rho \pi \int \delta^{2} d \delta=\frac{\rho \pi \delta^{3}}{3}$

Since the quantities $\rho, \pi, \Delta N$ are considered in the calculation of the distribution function on the linear dimensions, functions fs and fm can be reduced to these constants. Thus, to calculate the average on size and weight will have the following relations (8), (9):

$$
\begin{aligned}
& \Delta s_{i}=\delta_{i+1}^{3}-\delta_{i}^{3} \\
& \Delta m_{i}=\delta_{i+1}^{4}-\delta_{i}^{4}
\end{aligned}
$$

Expressions for calculating the average surface diameter and median diameter take the form (10) and (11), respectively:

$$
\begin{aligned}
& \delta_{20 i}=f n i \cdot \Delta s_{i} \\
& \delta_{30 i}=f n i \cdot \Delta m_{i}
\end{aligned}
$$

To calculate the Sauter average diameter the formula is accepted:

$\delta_{32}=\frac{\int_{\delta_{i}}^{\delta_{i+1}} f(\delta) \bar{\delta}_{l}^{3} d \delta}{\int_{\delta_{i}}^{\delta_{i+1}} f(\delta) \bar{\delta}_{\imath}^{2} d \delta}$

Considering all the previous conversion and reduction, as well as after simple mathematical transformations, it is easy to obtain an expression for calculating the Sauter average diameter of the following:

$\delta_{32}=\left(\delta_{30}\right)^{3} /\left(\delta_{20}\right)^{2}$

Similarly, other values of this function are calculated. After summation of the values of the size on the area was calculated distribution function of the size on the area:

$f_{S} 0-20=145.349$

The distribution function of the size by weight is calculated on the same principle. The mass of particles in the size range 0-20 microns was:

$$
\Delta m_{0-20}=0.00002^{4}-0^{4}=1.6 \cdot 10^{-19}
$$

$f_{M 0-20}=7,621$

The $9^{\text {th }}$ histogram, corresponding to the resonance frequency $(12.67 \mathrm{~Hz})$, shows predomination of fine fraction in 
the range 100-140 and 140-180 microns.

Knowing the values of the distribution function, we can calculate the average diameters on the linear dimension, space, mass and the average diameter of Sauter.

The average diameter on the linear dimension is:

$\delta_{10}=\frac{\int_{\delta_{i}}^{\delta_{i+1}} f(\delta) \bar{\delta}_{i} d \delta}{\int_{\delta_{i}}^{\delta_{i+1}} f(\delta) d \delta}$

where: $\bar{\delta}_{1}$ - average value for the interval, microns;

$f(\delta)$ - the distribution function of the linear dimension;

$\mathrm{d} \delta$ - the particle size of the respective fractions.

The average diameter on the linear dimension in the size range of $0-20$ microns is:

$\delta_{100-20}=5.098 \mu \mathrm{m}$.

The average diameter on the area in the size range of $0-20$ microns by the formula (10):

$\delta_{200-20}=2,039 \cdot 10^{-4} \mu \mathrm{m}$.

The average diameter by mass in the size range of $0-20$ microns by the formula (11):

$\delta_{300-20}=4.078 \cdot 10^{-9} \mu \mathrm{m}$.

The average diameter on the linear dimension, transform the formula and calculate the expression:

$\delta_{10}=\sum_{i} \delta_{10}=22,98 \mu \mathrm{m}$.

Considering all the previous conversion and reduction, as well as after simple mathematical transformations, it is easy to obtain an expression for calculating the average diameters $\delta_{20}$ and $\delta_{30}$ :

$$
\begin{aligned}
& \delta_{20}=\left(\frac{\Sigma_{i} \delta_{20}}{3}\right)^{1 / 2}=27,68 \mu \mathrm{m} . \\
& \delta_{30}=\left(\frac{\Sigma_{i} \delta_{30}}{4}\right)^{1 / 3}=34,15 \mu \mathrm{m} .
\end{aligned}
$$

Thus, the average Sauter diameter $\delta_{32}$ by the formula (13) is:

$\delta_{32}=51,97 \mu \mathrm{m}$.

An important characteristic of the particles' dispersion degree is their specific surface. The specific surface area determines the intensity of metabolic processes such as adsorption, extraction, dissolution taking place at the surface of the dispersed phase. Knowing the value of the Sauter average diameter, you can calculate the value of the specific surface area for our drops, corresponding to different frequencies and then build this relationship graphically.

$$
S_{\text {yд }}=\frac{S_{\mathrm{K}}}{V_{\mathrm{K}}}=\frac{6}{\delta_{32}}
$$

where: $S_{K}$ - the projected area of the drop;

VК - volume of the droplet;

$\delta_{32}-$ average droplet diameter of Sauter.

Thus, the values of drops' specific surface, corresponding to the frequencies at which the experiments were conducted, are the following:

Table 7: Table of values of the specific surface

\begin{tabular}{|c|c|}
\hline$f, \mathrm{~Hz}$ & Sspec, $1 / \mathrm{m}$ \\
\hline 11.1 & 22430 \\
\hline 12.035 & 30860 \\
\hline 12.67 & 115600 \\
\hline 14.183 & 58940 \\
\hline 14.818 & 80750 \\
\hline
\end{tabular}

The curve is constructed as a polygon. From this graph, it is clear that the resonant mode is the most beneficial to the mass transfer processes, as it facilitates the formation of the developed surface of phase contact and increases the intensity of mixing.

The ratio $\delta_{\max }$ to $\delta_{32}$ is presented in Table 8 . 


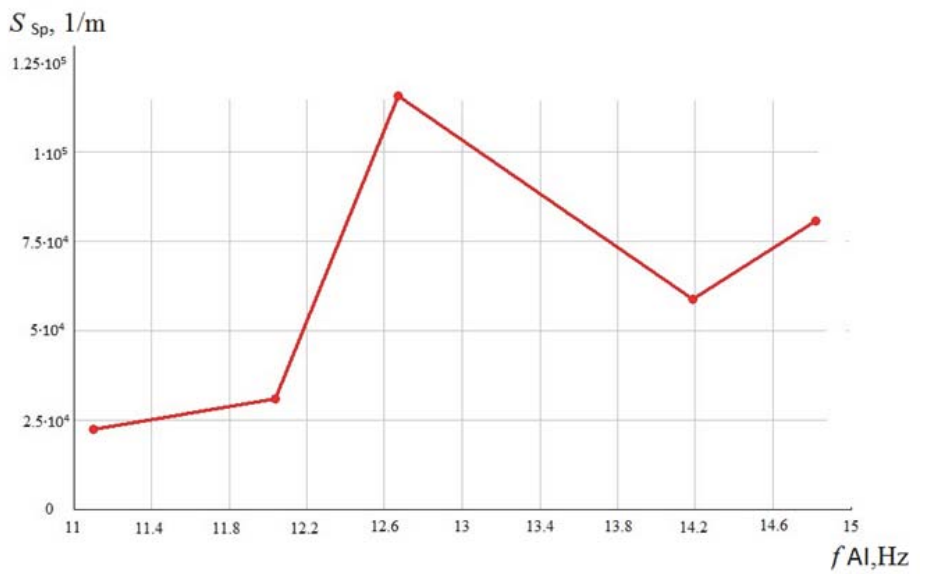

Figure 8: The dependence of the specific surface of the droplets from the stem's oscillation frequency

Table 8: The ratio of the maximum diameter to the average diameter of the Sauter

\begin{tabular}{|c|c|}
\hline$f, H z$ & $\delta_{\max } / \delta_{32}$ \\
\hline 11.115 & 1.279 \\
\hline 12.035 & 1.399 \\
\hline 12.67 & 3.083 \\
\hline 14.183 & 3.183 \\
\hline 14.818 & 5.249 \\
\hline
\end{tabular}

According to our theory, there is the possibility of the power measuring consumed for dispersing of dispersed droplets in a continuous phase, by applying the values of maximum diameter of drops samples on graph of diameters of the energy dissipation. This graph, shown in the book of Dolinskii and Ivanitskii (2008), compares the energy spends for the preparation of emulsions in conventional dispersants of different capacities, as well as dispersants, operating on the principle of discrete-pulsed energy input into the system. This graph is shown in Figure 9.

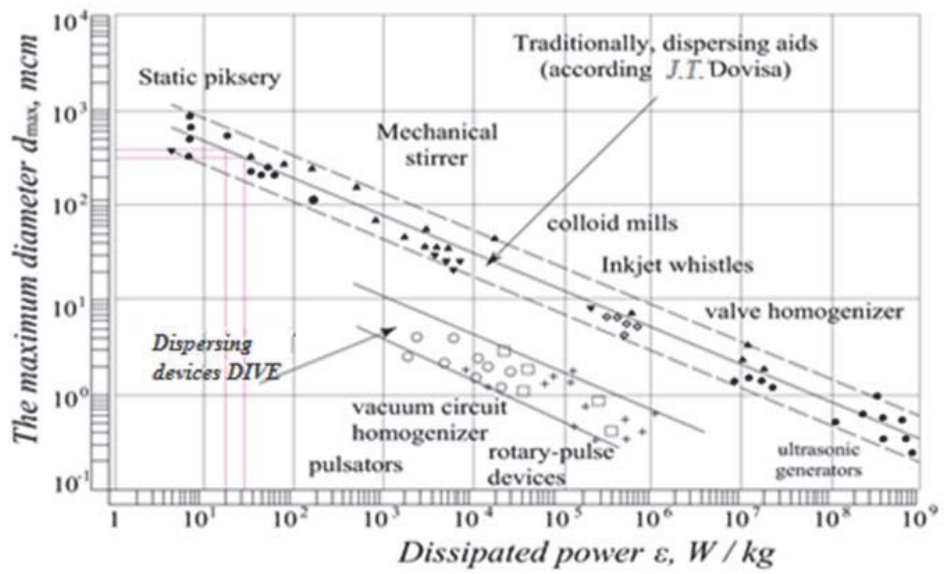

1-dissipated power at $\delta_{\max }=342 \mu \mathrm{m}(f=11,115 \mathrm{~Hz}) ; 2$ - dissipated power at $\delta_{\max }=272 \mu \mathrm{m}(f=12,035 \mathrm{~Hz}) ; 3$ - dissipated power at $\delta_{\max }=160 \mu \mathrm{m}(f=12,67 \mathrm{~Hz}) ; 4$ - dissipated power at $\delta_{\max }=324 \mu \mathrm{m}(f=14,183 \mathrm{~Hz}) ; 5$ - dissipated power at $\delta_{\max }=390 \mu \mathrm{m}(f=$ $14,818 \mathrm{~Hz})$.

Figure 9: Energy dissipation necessary to disperse of droplets in different devices 
For determining the dissipated power, HPRA was tentatively attributed to the group of conventional dispersing aids.

With the help of the Kompas 3D program the graph was processed, the points, corresponding to the dissipated power, were plotted. This power contributes to the fragmentation of drops to the investigated earlier diameter. Power N (W) is found as multiplication of dissipated power $(\mathrm{W} / \mathrm{kg}$ ) on the total weight of the emulsion (Mo $=1.15 \mathrm{~kg})$. Thus, the specified frequency range corresponds to the following power values is presented in Table 9.

Table 9: The results of the graphic processing of the droplet diameters

\begin{tabular}{|l|c|c|c|c|c|}
\hline The frequency of stock $\mathrm{f}, \mathrm{Hz}$ & 11.12 & 12.04 & 12.67 & 14.18 & 14.82 \\
\hline The diameter of the droplets $\delta_{\text {max }}$, micrometers & 342 & 272 & 160 & 324 & 390 \\
\hline Dissipated power $\varepsilon, W / k g$ & 11.8 & 14 & 111 & 12.2 & 10.9 \\
\hline Power, $\mathrm{Ngr}, \mathrm{W}$ & 13.57 & 16.1 & 127.65 & 14.03 & 12.535 \\
\hline
\end{tabular}

However, the power that was spent by system on mixing cannot be greater than that which is used to maintain the operation.

Thus was produced a graphical comparison of the power expended in crushing the oil droplets in water (Ngr, W) with electric power $\left(\mathrm{P}_{e}, \mathrm{~W}\right)$ and the power determined graphically with an adjustment factor $\mathrm{k}=0.76\left(\mathrm{~N}^{\prime} \mathrm{gr}, \mathrm{W}\right)$. Graphically, this is reflected on a Figure 15.

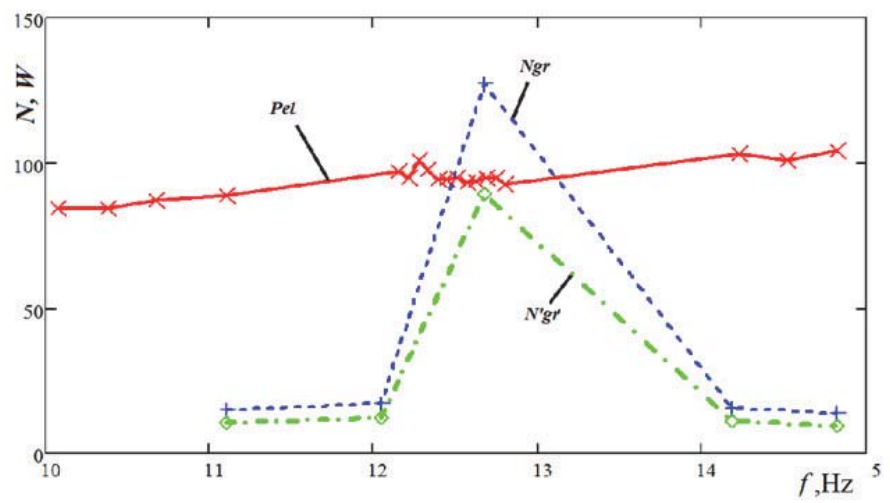

Figure 10: Comparison of electric power and power expended in the dispersion calculated graphically

Thus, all of our values of power will be reduced to the level of electrical power. High values of power indicate that during crushing of drops in the experiment GPRA prevails not only turbulent crushing mechanism, but the mechanisms of crushing, which are peculiar to the apparatuses with discrete pulse energy input to the system. Also worth noting is that most fully entered into the system power is dissipated in the resonance mode.

The power values consumed on the dispersion in the experimental setup of horizontal pulsation resonance apparatus are shown in Table 10.

Table 10: The power expended in the dispersion of oil droplets in water

\begin{tabular}{|l|c|c|c|c|c|}
\hline The frequency of stock $f, \mathrm{~Hz}$ & 11.115 & 12.035 & 12.67 & 14.183 & 14.818 \\
\hline The diameter of the droplets $\delta_{\max }$, micrometers & 342 & 272 & 160 & 324 & 390 \\
\hline Power $N, W$ & 13.57 & 16.1 & 127.65 & 14.03 & 12.535 \\
\hline Power $N$ 'gr, $W$ & 10.3 & 12.236 & 97.014 & 10.663 & 9.526 \\
\hline
\end{tabular}

As seen from Table 10, the resonant mode of the device contributes to more efficient crushing of drops. Moreover, from this graph is seen, that pre- and post-resonance frequencies not all power is introduced into the system. The ratio of dissipated power to electric increases from $11.6 \%$ to $94.1 \%$ (resonance), and then drops to $9.1 \%$. This once again proves the positive influence on the system of using a resonant mode, since power is introduced into the system more fully. 


\section{Conclusions}

The experiments revealed that the resonant mode of HPRA contributed for the most efficient crushing of the oil droplets, and thus had the most beneficial effect on the development of phase contact surface. For determining the intensity of metabolic processes occurring on the surface of the dispersed phase, was built dependence of the specific surface of the droplets on the frequency, which showed that the resonant mode enhanced the formation of the developed surface of phase contact.

Calculation of power required for droplets of sunflower oil dispersing in water showed that resonance mode of the apparatus contributes to more efficient crushing of drops. Besides it was established experimentally that the HPRA installation in the process involved not only the turbulent mechanism of drops crushing, but also the mechanisms inherent in devices with discrete pulse energy input. It has been observed that most of the power input into the system was spent on dispersion of oil droplets in aqueous medium, and the part that has been spent on the heating of system and the working environment was so small that quickly exit from the system through the support in the form of heat. The system simply counterbalanced its temperature to ambient temperature.

Pulsation mixing is more efficiently than mechanical, namely pulsating effect on work medium with a continuous liquid phase is a method of intensifying the process of mass transfer and increases economic performance, especially through resonance fluctuations. Since the oscillations of the fluid in the sections, arranged symmetrically to the pulsation chambers, are out of phase, and the fluid mass are equal, the dynamic load on the foundation is fully offset, which increases the reliability of the apparatus. Thus, along with a variable pressure in the fluid is realized good mixing in apparatus as a whole.

Summarizing all the above, we can conclude that the principles of generating oscillations realized in horizontal pulsating resonance apparatus have great potential for use in modern equipment. Examples of application are: 1) extraction processes and chemical reactions in the liquid-liquid systems, including biodiesel production, thin organic synthesis; 2) dissolution processes of solid particles, extraction, including dissolution of flocculants in water treatment processes; 3) purification of membranes in a membrane apparatus, including the apparatus described by Azimov, Abiev, Shakirov, Zhantasov (2014); 4) also possible imposition of fluctuations in the suspension in other types of water treatment equipment.

\section{References}

Abiev R.Sh. (2008) Pulsating devices of new generation - energy and resource conservation equipment of chemical production, Chemical Industry Today 4: 46-54.

Abiev R.Sh. (2012) Intensification of industrial wastewater treatment in the pulsating flow type apparatus, Izv. SPbSTI (TU) 13 (39): $82-$ 86.

Abiev R.Sh., Azimov A.M., Shakirov B.S. (2013) Ways to improve waste water treatment with the use of thin-layer settlers, Chemical Industry, 90 (6): 286-290.

Abiev R.Sh, Vasilev M.P., Doilnitsyn V.A. (2015) Investigation of the vacuum degassing water by means of a vortex jet apparatus, Izvestija SPbSTI (TU) 28 (54): 64-69.

Azimov A.M., Abiev R.Sh., Shakirov B.S., Zhantasov K.T. (2014) Clean water flows in the membrane type pulsation apparatus, Izvestija SPbSTI (TU), 23 (49): 62-65.

Azimov A.M., Abiev R.Sh., Zhantasov K.T., Sataeva L.M., Azimova N.B., Zerhatbek M.M. (2015) Modelling of pulsating membrane device for desalination of natural and industrial waters with periodic discharge of sludge thickening, Izestija of universities. Technology of textile industry 1 (355): 132-138.

Dolinskii A.A., Ivanitskii G.K., 2008 Heat and mass transfer and hydrodynamics in the vapor-liquid dispersion environment: thermophysical basics of discrete impulse energy input. Kiev: Naukova Dumka.

Ganiev R.F., Ukrainskii L.E., 2011. Nonlinear wave mechanics and technology. Wave and oscillatory phenomena in high-tech. M.: Institute comp. res.; SIC "Regular and chaotic dynamics".

Sarkisov P.D. (2000) Problems of energy and resource conservation in chemical engineering, petrochemical and biotechnology, Chemical Industry. 1: 20 - 27.

Yaroschik I.V., Abiev R.Sh. (2013) Study of efficiency of slurry separation with low density difference in a hydrocyclone with a diffuser, Chemical Industry, 90 (4): 185-189. 